nur, wie bisher meist angenommen, zur Befreiung in Haftstellen gebundener Elektronen führt, sondern daß sie außerdem die Elektronen-Aufnahmebereitschaft der Leuchtstellen oder die Zahl der aufnahmebereiten beeinflußt, wie noch kurz erläutert werden soll.

Ein im erregten Phosphor gespeicherter Energievorrat sei gegeben durch die Gesamtzahl $N=n_{A}$. $+n_{B^{*}}+\ldots \ldots$ ionisierter Aktivatorstörstellen (Leuchtstellen) $A^{*}, B^{*}, \ldots \ldots$ und die gleiche Zahl $N$ an Haftstellen gebundener Elektronen. Er verteilt sich bei der Rückkehr in den unerregten Zustand - also bei der Wiedervereinigung der Elektronen mit den Leuchtstellen - auf die Aktivatoren $A, B, \ldots \ldots$ einschließlich solcher Stellen, an denen die Rekombination ohne Strahlung vor sich geht. Diese Verteilung ist - das

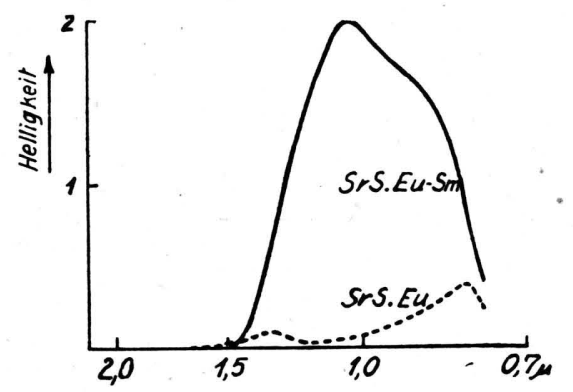

Abb. 1. Spektrale Verteilung der Ausleuchtung. Ordinate: Helligkeit des Emissionslichtes (Spektrum desselben: Z. Naturforschg. 1, 70 [1946], Abb. 8) beim - Auftreffen von Licht der auf der Abszisse angegebenen Wellenlänge. Die Kurven sind auf gleiche auffallende Strahlungsleistung pro $\mathrm{cm}^{2}$ reduziert.

zeigen die Versuche - beim Ausheizen anders als beim Ausleuchten. Die Teillichtsumme, die zu einem Aktivator $A$ gehört, hängt also sehr eng mit dem Anteil $n_{\boldsymbol{A}}$. der aktivatorspezifischen Leuchtstellen an der Gesamtzahl $N$ zusammen. Wenn das Größenverhältnis der Teillichtsummen beim Ausheizen und Ausleuchten verschieden ist, dann müssen unter der Wirkung des auslöschenden Lichtes Bewegungen der positiven Ladungen, d. h. Verschiebungen der Gleichgewichte

$$
A^{\cdot}+B \stackrel{\leftrightarrow}{\rightarrow} A+B^{\cdot}
$$

vorkommen ${ }^{5}$. Hierbei ist die ultrarote Eigenabsorption eines Aktivators wahrscheinlich von Einfluß, was in der spektralen Verteilung der Empfindlichkeit gegen ausleuchtendes Licht erkennbar werden müßte. Hierüber kann vorläufig folgendes gesagt werden.

Mißt man die spektrale Ausleuchtverteilung nach der Dahmsschen Methode ${ }^{6}$ an einem SrS.Eu-Phosphor, so stellt man zwei Maxima zwischen 0,75 und 1,5 $\mu$ fest (Abb. 1). Sensibilisiert man diesen Phosphor durch Zugabe von $\mathrm{Sm}^{7}$, so tritt, wie Abb. 1 zeigt, ein neues Empfindlichkeitsmaximum auf, welches mit dem Spektralgebiet starker Absorption des $\mathrm{Sm}+++(0,9$ bis $1,6 \mu$ ) ungefähr übereinstimmt ${ }^{8}$. Der Schluß, daß $\mathrm{Sm}$ - wie schon von $\mathrm{R}$. Tom a s che k vermutet - die Ursache für jenes Empfindlichkeitsmaximum ist, gewinnt dadurch an Wahrscheinlichkeit, daß die Ausleuchtverteilung des SrS.Ce-Sm die gleiche Form und Lage hat, während das Emissionsspektrum in beiden Fällen grundverschieden ist: SrS.Eu-Sm zeigt das Eu++-Spektrum, SrS.Ce-Sm das Ce+++-Spektrum. Übrigens besteht auch eine Ähnlichkeit im Temperaturverhalten der beiden Mischphosphore: Ausleuchtendes Licht wirkt bei beiden bei $-196^{\circ}$.C wesentlich schwächer als bei $+20^{\circ}$ C. Hingegen wirkt ausleuchtendes Licht auf den reinen SrS.Eu-Phosphor bei tieferen Temperaturen $\left(+78^{\circ} ;-196^{\circ} \mathrm{C}\right)$ sehr viel stärker als bei Zimmertemperatur. Ist die Auffassung von der Sm+++-Absorption als Ursache der spektralen Ausleuchtempfindlichkeit richtig, so sollte man erwarten, daß auch reines SrS.Sm sich diesbezüglich wie die Mischphosphore verhielte. Das ist nun zwar der Fall, doch strahlt SrS.Sm beim Ausleuchten schwach kontinuierlich statt mit dem Sm+++-Spektrum, weshalb man diese Beobachtung bei der bekannten Gefahr, selbst bei größter präparativer Sorgfalt, einen durch eine unbekannte Verunreinigung hervorgerufenen Effekt vor sich zu haben, zu sicheren Schlüssen noch nicht verwenden kann.

5 Wegen Einzelheiten muß auf künftige Veröffentlichungen von Hrn. Prof. W. S c h o t t ky verwiesen werden.

6 A. D a h m s, Ann. Physik 13, 425 [1904].

7 P. B r a u e ${ }^{2}$, S. 75.

8 Siehe z. B. G. R o s e n th a l, Physik. Z. 40, 508 [1939]. Die dort mitgeteilten Spektren von $\mathrm{Sm}+++$ wurden mit Hilfe einer Boraxperle aufgenommen. Der Vergleich mit den SrS-Phosphoren kann nur einen ungefähren Anhalt geben, da in Abhängigkeit vom Grundmaterial die Spektren etwas verschieblich und die relative Intensität ihrer Teilbanden sogar stark veränderlich sind.

\section{Über einige Salze des Indiums V. Uber die Indate ${ }^{1}$}

Von Fritz Enßlin und Siegfried Valentiner Aus dem Hauptlaboratorium Oker der Unterharzer Bergund Hüttenwerke G.m.b.H., Goslar, und dem Physikal. Institut der Bergakademie Clausthal

(Z. Naturforschg. 2 a, 239 [1947]; eingeg. am 2. Januar 1947)

Bericht über Herstellung und Kristallstrukturbestimmung einiger Indate, deren Eigenschaften, einschließlich Farbe und Feinbau, beschrieben werden.

1 Zusammenfassung der in Z. Naturforschg. 2b, Heft 1/2, S. 5 [1947] erschienenen Arbeit. 